\title{
Utilidad de la tomografía lineal para el reconocimiento de estructuras anatómicas
}

\section{Pedro Luiz de Carvalho' Odalício Vieira de Siqueira ${ }^{2}$ Elídia Mara dos Santos Carvalho $^{3}$ Clifford Alejandro Pedro Paulo Allen Lengua ${ }^{4}$ \\ Luiz Carlos Laureano da Rosa ${ }^{5}$ João Marcelo Ferreira de Medeiros $^{6}$}

Docente del Departamento de Odontologí. Universidad de Taubaté - UNITAU, São Paulo, Brasil. Universidad Universidad de Taubaté - UNITAU, São Paulo, Brasil. Cirujana Dentista.

Docente del Universidad Inca Garcilaso de la Vega Lima, Peru.

Docente del estadistica y Investiagador do NUPES Núcleo de Pesquisas Econômico-Sociais da UNITAU. Universidad de Taubaté - UNITAU, São Paulo, Brasil.

'Docente del Curso de Pós Graduacion em Odontología Especialidad Endodoncia Universidad de Taubaté - UNITAU, São Paulo, Brasil.

\section{Correspondencia}

Pedro Luiz de Carvalho.

Rua dos Jacarandás, 3210 - Jardim Santa Helena CEP 12.916-423

Braganca Paulista - SP, Brasi

Telefono: $3329146 / 98440005$

e-mail: pedrolc@unitau.br

Recibido : 4 de setiembre del 2008

Aceptado : 30 de noviembre del 2008
Carvalho PL, Siqueira OV, Santos Carvalho EM, Allen Lengua CAPP, Rosa LCL, Medeiros JMF Utilidad de la tomografía lineal para el reconocimiento de estructuras anatómicas. Rev Estomatol Herediana. 2008; 18(2):99-104.

\section{RESUMEN}

El objetivo de este trabajo fue evaluar la imagen de las estructuras anatomicas de exámenes tomográficos transversales obtenidos del sector de molares inferiores, en el equipo Orthophos Plus/CD. En diez mandíbulas de adultos se obtuvieron cortes tomográficos P19 del lado derecho y P20 del lado izquierdo. A partir de la imagen central, cinco examinadores calibrados realizaron la evaluación de las imágenes, separadamente para identificar las siguientes estructuras anatómicas: cortical ósea alveolar (COA), cortical ósea lingual, (COL), cortical ósea vestibular (COV), canal mandibular (CM) y bases de la mandíbula (BM). Para la evaluación de las imagenes de las estructuras anatómicas se utilizaron los siguientes parámetros: (1) cuándo las imagenes de las estructura anatómicas no estaban presente o no visible, (2) cuándo la imagen de las estructuras anatómicas presentaban poco visible y (3) cuándo la imagen de las estructuras anatómicas estaban visibles. Los datos fueron agrupados en tablas y analizados por la prueba estadística de Kappa. Se calculó además la sensibilidad, especificidad, valores predictivos positivos-negativos, y experiencia de cada examinador. Se concluyó que la imagen tomográfica de las estructuras anatómicas: cortical ósea alveolar, cortical ósea lingual, cortical ósea vestibular, canal mandibular y base de la mandíbula pueden ser idientificadas. Sin embargo, a pesar de la reproductibilidad los resultados tomográficos obtenidos en el equipo de rayos X Ortophos algunas veces no son satisfactorios para el sector de molares inferiores, por esta razón se recomienda la asociación con otro examen radiográfico.

Palabras clave: TOMOGRAFÍA POR RAYOS X / DIAGNÓSTICO POR IMAGEN.

\section{Efficacy of lineal tomography to detect anatomical structures} ABSTRACT

The purpose of this study was to evaluate anatomic structure image of cross-sectional tomographic examination obtained in the lower molar area, with the Orthophos Plus/CD device. For this examination 10 adult mandibles were utilized to perform the cross-sectional tomographic examination with programs for tomographic slice P19 (right side) and P20 (left side). From the images obtained, using central image, five qualified examiners performed the evaluation of such images; who, separately, obtained the identification of the following anatomical structures: cortical bone on crest of alveolar ridge (CBA), lingual cortical plate (LCP), buccal cortical plate (BCP), inferior alveolar canal (IAC) and inferior cortical plate (ICP). In the images evaluation of anatomical structures the following scores were used: (1) when anatomical structures images were not present or were not visible, (2) when anatomical structures images presented themselves insufficiently visible, and (3) when anatomical structures images were visible. Data were sorted in charts and analyzed through Kappa's statistical test at significance level of $5 \%$. It was also calculated sensitivity, specificity, positive and negative predictive values and each examiner's accuracy. It was concluded that tomographic image of anatomical structures: cortical bone on crest of alveolar ridge, lingual cortical plate inferior alveolar canal and inferior cortical plate are observed in cross-sectional topographies. However, despite the reproducibility, sometimes tomographic results, obtained through Ortophos x-rays are not satisfactory for lower molar area; thus, it is recommended that there be an association with another radiographic examination.

Key words: X-RAY TOMOGRAPHY / DIAGNOSTIC IMAGING

\section{Introducción}

La tomografia es un método de examen radiográfico en el cuál una "tajada", o corte de una estructura del organismo se obtiene en un plano predeterminado. En la tomografia, la fuente de rayos $\mathrm{X}$ se mueve en una dirección, mientras el receptor de imagen se mueve en dirección opuesta. La fuente productora de rayos $\mathrm{X}$ y el receptor de imagen se mueven simultáneamente en relación constante, que es mantenida por un sistema de conexión que gira alrededor de un eje, en el plano de corte a ser proyectado.

La evaluación radiográfica proporciona al cirujano dentista información sobre la cantidad de hueso presente, la calidad ósea disponible y la localización de estructuras anatómicas, factores esenciales para la realización de procedimientos quirúrgicos (1-8). No obstante, los métodos de exámenes a través de imágenes desde las periapicales hasta las panorámicas, no registrando imágenes en plano coronal o frontal. A pesar de eso, la radiografía panorámica es el primer paso en la evaluación de los tejidos duros de un paciente dentado o desdentado (9).

Las tomografías permiten efectuar una representación tridimensional de los diferentes sectores de los maxilares superior e inferior, $\mathrm{y}$ algunos equipos de rayos $\mathrm{X}$ panorámicos presentan programas para esa finalidad $(10,11)$. 
En el sector molares inferiores podemos deparar con muchos problemas patológicos y/o quirúrgicos, así cuanto más informaciones obtengamos, mejor será el manejo del tratamiento (12). Independiente del problema patológico o quirúrgico, aparentemente simple, se debe realizar un examen clínico y un estudio radiográfico con la finalidad visualizar totalmente el área adyacente al problema central.

El trabajo puede perfeccionarse con el uso del equipo rayos $\mathrm{X}$ Orthophos Plus/CD, de la SiemensSirona, en la obtención de imagenes transversales en procedimientos quirúrgicos. De suerte que, la identificación de probables procesos que puedan adversamente afectar su éxito, solamente el imágenes sagitales y coronales posibilitan la adecuada determinación del área quirúrgica.

Por medio de las informaciones de los exámenes tomográficos obtenidos el equipo Orthophos, de marca Siemens-Sirona, modelo Plus/CD, el objetivo del estudio fue evaluar la imagen de las estructuras anatómicas en examen tomográficos transversales obtenidos del sector molares inferiores.

\section{Material y métodos}

El proyecto fue aprobado por Comité de Ética e Investigación de la Universidad de Taubaté, protocolo de aprobación $\mathrm{N}^{\circ} 0087 / 07$, de acuerdo con la Resolución 196/96 del Comité Nacional de Salud/Departamento de Salud.

Se utilizaron en esa investigación 10 mandíbulas de adultos, pertenecientes al Laboratorio de Anatomía del Instituto Básico de Biociencias de Universidad de Taubaté. El tamaño de la muestra fue determinado por la cantidad disponibles de mandíbulas para la realización de la

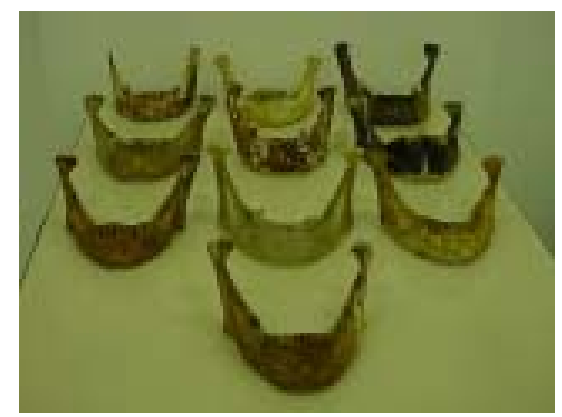

Fig. 1. Mandíbulas utilizadas.

investigación (Fig. 1).

Se emplearon películas de marca Kodak, tipo T-mat T, de 15x30 centímetros (Kodak Brasileira Comércio e Indústria Ltda.), que fueron acondicionados en chasis rígido junto con dos placas intensificadoras (Kodak - tipo Lanex regular).

Como fuente productora de rayos $\mathrm{X}$, se utilizó el equipo panorámico Orthophos, de marca SiemensSirona, modelo Plus/CD (Alemania), que operó con Kilovoltaje de $60 \mathrm{kVp}$, miliamperaje de $16 \mathrm{~mA}$, tiempo de exposición de 7,3 segundos (Fig. 2). Además, se utilizó filtraje adicional para haz de rayos $\mathrm{X}$, formada por una laminilla de plomo de una película radiográfica oclusal doblada al medio y posicionada con cinta adhesiva a frente del diafragma del equipo.

Las mandíbulas fueron preparadas con la adaptación de una esfera metálica de 5 milímetros de diámetro, en sector de terceros molares o cerca a ello, posicionada al centro del reborde alveolar, parte superior o sobre el diente molar más posterior presente (2) (Fig. 3).

Para la realización del examen tomográfico transversal, se utilizaron los programas para cortes tomográficos P19 (examen de lado derecho) y P20 (examen de lado izquierdo), cuyo factor de ampliación es 1,37; grosor de los cortes de 3,5 $\mathrm{mm}$ y la distancia entre los cortes de 2,5 a 3 milímetros (13).

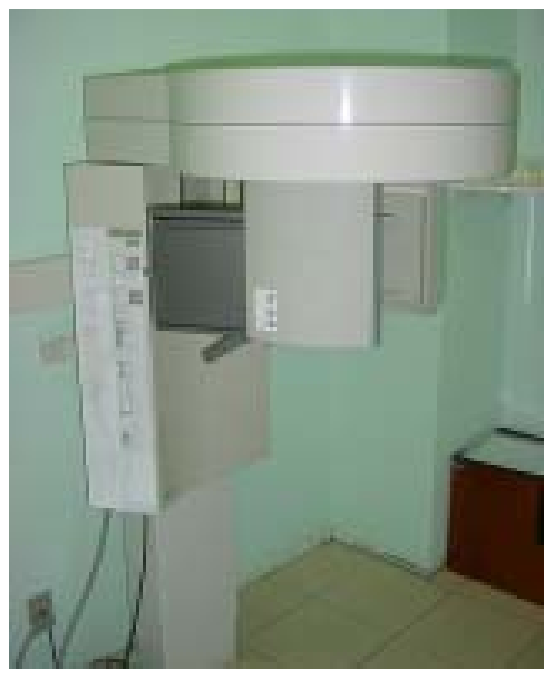

Fig. 2. Equipo de rayos $X$ panorámico Orthophos, modelo Plus/CD.

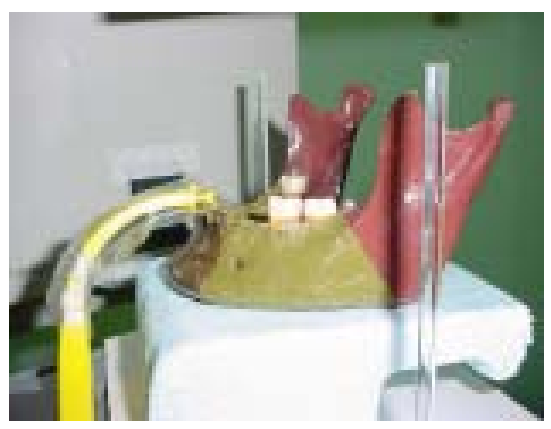

Fig. 3. Mandíbulas con adaptación de las esferas metálicas.

La estándarización del posicionamiento de las mandíbulas se cumplió rigurosamente, dado que es un factor primordial para la obtención de los exámenes tomográficos. De tal manera que se ajustó la inclinación de la mandíbula utilizando el foco luminoso horizontal, de tal forma que la base de la mandíbula quedó paralela al plano horizontal (proporcionado por el foco luminoso). A continuación la esfera se ubicó en la dirección exacta del bastón de marcación del lado a ser expuesto, siendo que los dos bastones quedaron alineados entre si.

Las películas fueron procesadas según el método tiempo-temperatura con soluciones Kodak.

A partir de la obtención de las imágenes, utilizándose la imagen 
central, cinco examinadores capacitados realizaron la evaluación estas, separadamente realizaron la identificación de las siguientes estructuras anatómicas: cortical ósea alveolar (COA), cortical ósea lingual (COL), cortical ósea vestibular (COV), canal mandibular (CM) y base de la mandíbula (BM). Para la evaluación de las imágenes de las estructuras anatómicas se utilizaron los siguientes parámetros: (1) cuándo las imágenes de las estructuras no estaban presentes o no visible, (2) cuándo la imagen de las estructuras anatómicas se presen- taban poco visibles y (3) cuándo la imagen de las estructuras anatómicas estaban visibles (Fig. 4,5,6).

Los resultados obtenidos fueron sometidos al análisis y estadística utilizando el software BIOESTAT 3,0. Fueron calculadas las reproducibilidades interexaminadores de los exámenes tomográficos, utilizando el test de Kappa al nivel de significancia de $5 \%$. Los valores de Kappa (K) fueron calculados para concordancia inter-examinadores. Un valor $\mathrm{K}$ menor de 0 fue considerado sin concordancia; de 0 a 0,19 débil concordancia; de 0,20 a 0,39 regular con- bula no fue posible calcular, pues solamente ocurrió una de las categorías de evaluación.

Precisión diagnóstica de las estructuras

La precisión diagnóstica de los exámenes tomográficos en la predilección de las estructuras anatómicas son presentadas en las tablas 2 , $3,4,5$ y 6 .

\section{Discusión}

La tomografía lineal como recurso diagnóstico en odontología fue estudiada por Clark et al. (1), Stella y

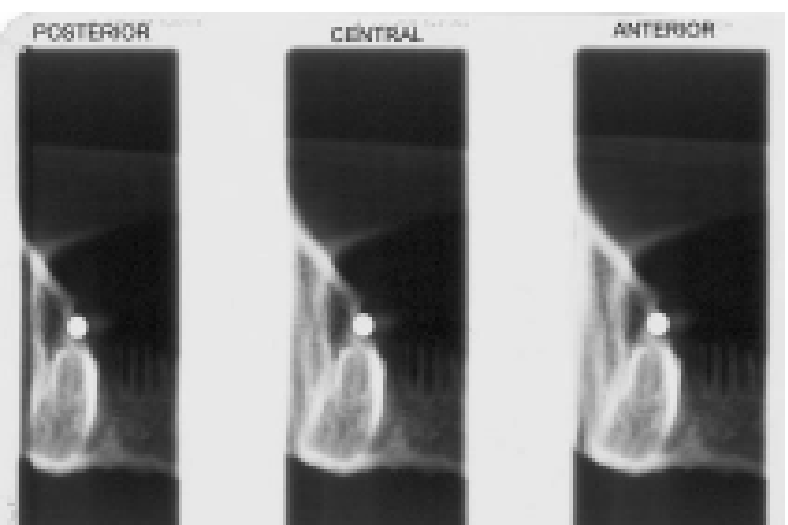

Fig. 4. Resultado tomográfico obtenido utilizando el Programa P19 de la mandíbula 9.

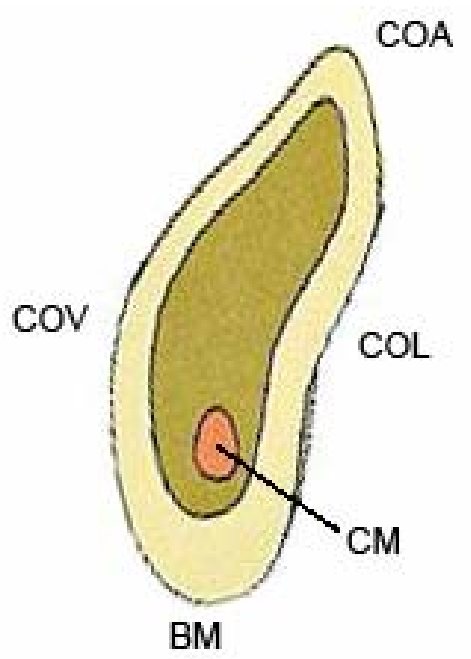

Fig. 6. Diseño esquemático de las estructuras anatómicas. Cortical ósea alveolar (COA), cortical ósea lingual (COL), cortical ósea vestibular (COV), canal mandibular (CM) y base de la mandíbula (BM). cordancia; de 0,40 a 0,59 aceptable concordancia; de 0,60 a 0,79 buena concordancia; y de 0,80 a 1,00 excelente concordancia (14). Se calculó también la sensibilidad, especificidad, valores predictivos positivosnegativos y experiencia de cada examinador.

\section{Resultados}

Reproductibilidad inter-examinador

Las reproductibilidades inter-examinadores para la imagen de la cortical ósea alveolar, cortical ósea lingual, cortical ósea vestibular y canal mandibular fueron de 0,48 ; 0,$38 ; 0,58$ y 0,54 respectivamente (Tabla 1). Sin embargo, la imagen de las estructura base de la mandí-
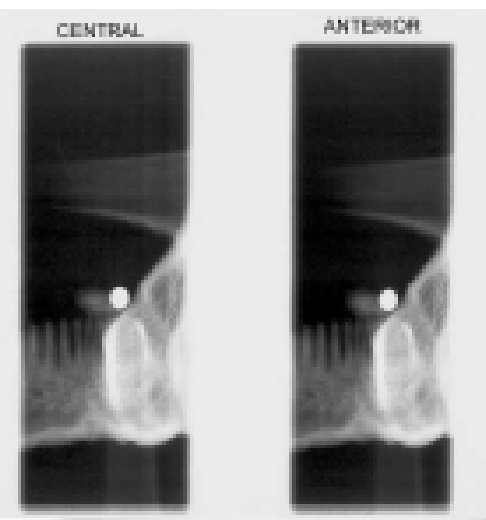

Fig. 5. Resultado tomográfico obtenido utilizando el Programa P20 de la mandíbula 9.

Tharanon (2), Kassembaum et al. (3), Bolin y Eliasson (5), Sonick et al. (6), Monahan y Furkart (8), Kassebaum et al. (9), Bell (12), Sethi (15), Silverstein et al. (16) y Butterfield et al. (17). En esos estudios se concluyeron que las imágenes tomográficas de las regiones o secciones del hueso proporcionan visualización sin sobreposición de las imágenes de estructuras anatómicas, reproduciendo con exactitud la imagen no obtenida por las técnicas radiográficas convencionales.

Debido a la difusión de las tomografías, las imágenes transversales están convirtiéndose fácilmente disponibles en odontología. Según Abrahms (4), Frederiksen (7), 
Tabla 1. Valores de Kappa, p-valor e intervalos de confianza de las estructuras anatómicas.

\begin{tabular}{|c|c|c|c|c|}
\hline \multirow{2}{*}{$\begin{array}{l}\text { Estructura } \\
\text { anatómica }\end{array}$} & \multirow[t]{2}{*}{ Kappa } & \multirow[t]{2}{*}{$\mathrm{P}$} & \multicolumn{2}{|c|}{ Intervalo de confianza } \\
\hline & & & Inferior & Superior \\
\hline$\overline{\mathrm{COA}}$ & 0,48 & $<0,001$ & 0,381 & 0,583 \\
\hline $\mathrm{COL}$ & 0,38 & $<0,001$ & 0,254 & 0,513 \\
\hline $\mathrm{COV}$ & 0,58 & $<0,001$ & 0,481 & 0,677 \\
\hline $\mathrm{CM}$ & 0,54 & $<0,001$ & 0,436 & 0,644 \\
\hline
\end{tabular}

COA: cortical ósea alveolar. COL: cortical ósea lingual. COV: cortical ósea vestibular. CM: canal mandibular. BM: base de la mandíbula.

Tabla 3. Precisión diagnóstica (\%) de la imagen tomográfica en la evaluación de la cortical ósea lingual.

\begin{tabular}{llrrrr}
\hline Ex & S & Es & VPP & VPN & Ea \\
\hline 1 & 60 & 50 & 100 & 0 & 60 \\
2 & 66,67 & 100 & 100 & 25 & 70 \\
3 & 75 & 50 & 100 & 0 & 75 \\
4 & 100 & 0 & 10 & 100 & 90 \\
5 & 75 & 50 & 100 & 0 & 75 \\
\hline
\end{tabular}

Ex: examinador. S: sensibilidad. Es: especificidad. VPP: valor predictivo positivo. VPN: valor predictivo negativo. Ea: exactitud.

Tabla 5. Precisión diagnóstica (\%) de la imagen tomográfica en evaluación del canal mandibular.

\begin{tabular}{llllll}
\hline Ex & S & Es & VPP & VPN & Ea \\
\hline 1 & 8,33 & 100 & 100 & 42,11 & 45 \\
2 & 30,77 & 100 & 100 & 43,75 & 55 \\
3 & 20 & 100 & 100 & 25 & 36,84 \\
4 & 46,15 & 100 & 100 & 50 & 65 \\
5 & 8,33 & 100 & 100 & 42,11 & 45 \\
\hline
\end{tabular}

Ex: examinador. S: sensibilidad. Es: especificidad. VPP: valor predictivo positivo. VPN: valor predictivo negativo. Ea: exactitud.
Tabla 2. Precisión diagnóstica (\%) de la imagen tomográfica en la evaluación de la cortical ósea alveolar.

\begin{tabular}{llllll}
\hline Ex & S & Es & VPP & VPN & Ea \\
\hline 1 & 66,67 & 100 & 100 & 66,67 & 80 \\
2 & 64,29 & 100 & 100 & 54,55 & 75 \\
3 & 65 & 50 & 100 & 0 & 65 \\
4 & 50 & 100 & 100 & 18,18 & 55 \\
5 & 60 & 100 & 100 & 45,45 & 70 \\
\hline
\end{tabular}

Ex: examinador. S: sensibilidad. Es: especificidad. VPP: valor predictivo positivo. VPN: valor predictivo negativo. Ea: exactitud.

Tabla 4. Precisón diagnóstica (\%) de la imagen tomográfica en la evaluación de la cortical ósea.

\begin{tabular}{llllll}
\hline Ex & S & Es & VPP & VPN & Ea \\
\hline 1 & 58,33 & 100 & 100 & 61,54 & 75 \\
2 & 58,33 & 100 & 100 & 61,54 & 75 \\
3 & 43,75 & 100 & 100 & 30,77 & 55 \\
4 & 46,67 & 100 & 100 & 38,46 & 60 \\
5 & 46,67 & 100 & 100 & 38,46 & 60 \\
\hline
\end{tabular}

Ex: examinador. S: sensibilidad. Es: especificidad. VPP: valor predictivo positivo. VPN: valor predictivo negativo. Ea: exactitud.

Tabla 6. Precisión diagnóstica (\%) de la imagen tomográfica en la evaluación de Base de la mandíbula.

\begin{tabular}{llllll}
\hline Ex & S & Es & VPP & VPN & Ea \\
\hline 1 & 100 & 50 & 100 & 50 & 100 \\
2 & 100 & 50 & 100 & 50 & 100 \\
3 & 100 & 50 & 100 & 50 & 100 \\
4 & 100 & 50 & 100 & 50 & 100 \\
5 & 100 & 50 & 100 & 50 & 100 \\
\hline
\end{tabular}

Ex: examinador. S: sensibilidad. Es: especificidad. VPP: valor predictivo positivo. VPN: valor predictivo negativo. Ea: exactitud.
Kassebaum et al. (9), Thunty (10), Pasler y Visser (11), las ventajas principales de la tomografía son la definición espacial y la baja dosis de radiación. Así, se ejecutó un estudio del sector de molares inferiores para eso, se utilizó el programa P19 y P20 del equipo Ortophos de la casa Siemens que posee ampliación de $37 \%$ y puede proporcionar imágenes transversales con buena resolución. Con este instrumento, el presente estudio muestra que a pesar de algunas imágenes interferentes fue posible identificar las estructuras anatómicas.

La reproductibilidad inter-examinador indicó que los exámenes tomográficos transversales, obtenidos en el equipo Orthophos puedan ser indicados para el sector de molares inferiores. Sin embargo, notese que los examinadores presentaron dificultades en identificación de algunas estructuras anatómicas. Las dificultades encontradas pueden relacionarse con la calidad del examen tomográfico, imagen distorsionada de estructuras vecinas presentes, que diferencia de otros resultados tomográficos obtenidos por otros equipos específicos. Asociado a ese problema, se debe considerar que se utilizó mandíbulas aislada de patrón óseo. No obstante, para intentar mejorar el resultado, visando una estándarización, se utilizó filtración adicional para mantener la calidad de la imagen de los exámenes obtenidos (3). En ese punto, Abrahms (4) mencionó que la tomografía lineal es hecha con una grande distancia entre los cortes y proporciona imágenes con poca de- finición y contraste, por consiguiente convierte difícil la medición de estructuras.

La imagen tomográfica del canal mandibular presentó valores bajos para la sensibilidad, siendo esos valores estadísticamente significativos cuando fueron comparados con las imágenes de las otras estructuras. Esos valores sugieren poca fidelidad de ese examen tomográfico en la evaluación de esa estructura.

A pesar de la busqueda por métodos de alta sensibilidad para exámenes de diagnóstico por medio de la imagen, la alta especificidad evita la realización de examen de diagnóstico por medio de la imagen necesaria, que podría significar reducción de costos, baja dosis de radiación recibida por el paciente.

Las estructuras anatómicas pre- 
sentaron altos valores predictivos positivos. Esto implica en una reducción del porcentaje de resultados falso-negativos, evitando errores en el diagnóstico durante la evaluación del examen y según Butterfield et al. (17) las tomografías lineales son limitadas en la evaluación preoperatoria quirúrgica.

Por el análisis de los examinadores, se observó que el resultado obtenido en equipo Orthophos puede ser empleado en la evaluación del sector de molares inferiores, pero no debe ser utilizado de forma aislada, asociado a otro examen radiográfico que proporcione otro plano y de acuerdo con Silverstein et al. (16) otros exámenes permiten un mejor diagnóstico y plano de tratamiento al profesional. Además, se recomienda reciclajes de los exámenes por medio de la imagen, para que sea evaluado si el método central con sus combinaciones serán para la finalidad quirúrgica suficientes o no.

Aún, el examen tomográfico transversal obtenido con el equipo Orthophos podrá ser útil, para las situaciones donde se desee evaluar calidad y cantidad de hueso disponible, por lo tanto para tal finalidad se recomienda que el paciente sea desdentado posterior. Pero no solamente eso, el examen estudiado requiere pequeña exposición de radiación para su obtención, en relación a otros métodos como panorámico, tomografía computada, de ser también de bajo costo $(1,3,9)$.

El estudio demostró que cuando cinco examinadores experimentados evalúan una imagen, esta tarea no puede ser exactamente ser realizada. Estos resultados son atribuidos a la inestabilidad dimensional de la tomografía lineal, que contribuye en producir imágenes distorsidas de la arcada mandibular, así comprometiendo a exactitud de las informacio- nes necesarias para el sector de molares inferiores. Es de forma imperativa que los clínicos conozcan las limitaciones físicas del sistema tomográfico lineal, y sus efectos adversos potenciales, la calidad y la exactitud radiográfica de la imagen antes de usarlo en procedimientos clínicos.

Finalizando, la tomografía lineal obtenida en el equipo de rayos $\mathrm{X}$ Orthophos es controversial, pues algunas veces el examen no es satisfactorio para el sector deseado. Bajo ese aspecto, los profesionales de acuerdo su criterio de elección tiene hoy a la disposición una amplia variedad de posibilidades de examen de diagnóstico radiográfico para incrementar su plan de tratamiento que, en un pasado aún reciente, era completamente inimaginable.

\section{Conclusiones}

Basado en los resultados la imagen tomográfica de las estructuras anatómicas: cortical ósea alveolar, cortical ósea lingual, cortical ósea vestibular, canal mandibular y base de la mandíbula son observadas en las tomografías transversal. Sin embargo, a pesar de la reproductibilidad los resultados tomográficos obtenidos en el equipo de rayos $\mathrm{X}$ Ortophos algunas veces no son satisfactorios para el sector de molares inferiores, así se recomienda su asociación a otro examen radiográfico.

\section{Referencias bibliográficas}

1. Clark DE, Danforth RA, Barnes RW, Burtch ML. Radiation absorbed from dental implant radiography: a comparison of linear tomography, CT scan, and panoramic and intra-oral techniques. J Oral Implantol. 1990; 16(3):156-64.

2. Stella JP, Tharanon W. A Precise Radiographic Method to De- termine the Location of the Inferior Alveolar Canal in the Posterior Edentulous Mandible: Implications for Dental Implants. Part 2: Clinical Application. Int J Oral Maxillofac Implants. 1990; 5(1):23-9.

3. Kassebaum DK, Nummikoski PV, Triplett RG, Langlais RP. Cross-sectional radiography for implant site assessment. Oral Surg Oral Med Oral Pathol. 1990; 70(5):674-8.

4. Abrahams JJ. The role of diagnostic imaging in dental implantology. Radiol Clin North Am. 1993; 31(1):163-80.

5. Bolin A, Eliasson S. Panoramic and tomographic dimensional determinations for maxillary osseointegrated implants. Comparison of the morphologic information potential of two and three dimensional radiographic systems. Swed Dent J. 1995; 19(1-2):65-71.

6. Sonick M, Abrahams J, Faiella RA. A comparison of the accuracy of periapical panoramic and computerized tomographic radiograph in locating the mandibular canal. Int J Oral Maxillofac Implants. 1994; 9(4):455-60.

7. Frederiksen NL. Diagnostic imaging in dental implantology. Oral Surg Oral Med Oral Pathol Oral Radiol Endod. 1995; 80(5):540-54.

8. Monahan R, Furkart AJ. Technical note. Sagittal tomography as an adjunct to cross-sectional evaluation of select implant sites. Dentomaxillofac Radiol. 1996; 25(5):298-301.

9. Kassebaum DK, Stoller NH, Goshorn BI. Radiographic techniques for presurgical assessment of dental implant 
sites. Gen Dent. 1992; 40(6):5025, 509-10.

10. Thunthy KH. Interrelationship between cross-sectional and sagittal imaging in computerassisted dental implant

tomography. Dentomaxillofac Radiol. 2000; 29(2):65-9.

11. Pasler PA, Visser H. Radiologia odontológica. Texto e atlas. Porto Alegre: Artmed; 2006.

12.Bell GW. Use of dental panoramic tomographs to predict the relation between mandibular third molar teeth and the inferior alveolar nerve. Radiological and surgical findings, and clinical outcome. Br J Oral Maxillofac Surg. 2004; 42(1):21-7.

13. SIEMENS. Orthophos CD. Unit manual. Bensheim:Germany; 1989.

14.Landis JR, Koch GG. The measurement of observer agreement for categorical data. Biometrics. 1977; 33(1):159-74.

15. Sethi A. Precise site location for implants using CT scans: a technical note. Int $J$ Oral Maxillofac Implants. 1993;
8(4):433-8.

16. Silverstein LH, Melkonian RW, Kurtzman D, Garnick JJ, Lefkove MD. Linear tomography in conjunction with pantomography in the assessment of dental implant recipient sites. J Oral Implantol. 1994; 20(2):111-7.

17. Butterfield KJ, Dagenais M, Clokie C. Linear tomography's clinical accuracy and validity for presurgical dental implant analysis. Oral Surg Oral Med Oral Pathol Oral Radiol Endod. 1997; 84(2):203-9. 